\title{
Some results on vanishing coefficients in infinite product expansions
}

\author{
NAYANDEEP DEKA BARUAH and MANDEEP KAUR
}

Abstract. Recently, M. D. Hirschhorn proved that, if

$$
\sum_{n=0}^{\infty} a_{n} q^{n}:=\left(-q,-q^{4} ; q^{5}\right)_{\infty}\left(q, q^{9} ; q^{10}\right)_{\infty}^{3} \quad \text { and } \quad \sum_{n=0}^{\infty} b_{n} q^{n}:=\left(-q^{2},-q^{3} ; q^{5}\right)_{\infty}\left(q^{3}, q^{7} ; q^{10}\right)_{\infty}^{3}
$$

then $a_{5 n+2}=a_{5 n+4}=0$ and $b_{5 n+1}=b_{5 n+4}=0$. Motivated by the work of Hirschhorn, D. Tang proved some comparable results including the following:

If

$$
\sum_{n=0}^{\infty} c_{n} q^{n}:=\left(-q,-q^{4} ; q^{5}\right)_{\infty}^{3}\left(q^{3}, q^{7} ; q^{10}\right)_{\infty} \quad \text { and } \quad \sum_{n=0}^{\infty} d_{n} q^{n}:=\left(-q^{2},-q^{3} ; q^{5}\right)_{\infty}^{3}\left(q, q^{9} ; q^{10}\right)_{\infty},
$$

then

$c_{5 n+3}=c_{5 n+4}=0$ and $d_{5 n+3}=d_{5 n+4}=0$.

In this paper, we prove that

$$
\begin{aligned}
a_{5 n} & =b_{5 n+2}, a_{5 n+1}=b_{5 n+3}, a_{5 n+2}=b_{5 n+4}, a_{5 n-1}=b_{5 n+1}, \\
c_{5 n+3} & =d_{5 n+3}, c_{5 n+4}=d_{5 n+4}, c_{5 n}=d_{5 n}, c_{5 n+2}=d_{5 n+2}, \text { and } c_{5 n+1}>d_{5 n+1} .
\end{aligned}
$$

We also record some other comparable results not listed by Tang.

Key Words: $q$-series expansions; infinite $q$-products; Jacobi's triple product identity, vanishing coefficients.

2010 Mathematical Reviews Classification Numbers: Primary 33D15; Secondary 11F33.

\section{Introduction}

For complex numbers $a$ and $q$, with $|q|<1$, we define

$$
(a ; q)_{\infty}:=\prod_{k=0}^{\infty}\left(1-a q^{k}\right)
$$

and

$$
\left(a_{1}, a_{2}, \ldots, a_{n} ; q\right)_{\infty}:=\left(a_{1} ; q\right)_{\infty}\left(a_{2} ; q\right)_{\infty} \cdots\left(a_{n} ; q\right)_{\infty}
$$

In this paper we prove some new results on vanishing coefficients in the series expansions of certain infinite $q$-products. In the following few paragraphs we review the work done on this topic by the previous authors.

In 1978, Richmond and Szekeres [7] proved that if

$$
\sum_{n=0}^{\infty} \alpha_{n} q^{n}:=\frac{\left(q^{3}, q^{5} ; q^{8}\right)_{\infty}}{\left(q, q^{7} ; q^{8}\right)_{\infty}} \text { and } \sum_{n=0}^{\infty} \beta_{n} q^{n}:=\frac{\left(q, q^{7} ; q^{8}\right)_{\infty}}{\left(q^{3}, q^{5} ; q^{8}\right)_{\infty}}
$$


then the coefficients $\alpha_{4 n+3}$ and $\beta_{4 n+2}$ always vanish. They also conjectured that if

$$
\sum_{n=0}^{\infty} \gamma_{n} q^{n}:=\frac{\left(q^{5}, q^{7} ; q^{12}\right)_{\infty}}{\left(q, q^{11} ; q^{12}\right)_{\infty}} \text { and } \sum_{n=0}^{\infty} \delta_{n} q^{n}:=\frac{\left(q, q^{11} ; q^{12}\right)_{\infty}}{\left(q^{5}, q^{7} ; q^{12}\right)_{\infty}}
$$

then $\gamma_{6 n+5}$ and $\delta_{6 n+3}$ vanish.

In [2], Andrews and Bressoud proved the following general theorem, which contains the results of Richmond and Szekeres as special cases.

Theorem 1.1. (Andrews and Bressoud) If $1 \leq r<k$ are relatively prime integers of opposite parity and

$$
\frac{\left(q^{r}, q^{2 k-r} ; q^{2 k}\right)_{\infty}}{\left(q^{k-r}, q^{k+r} ; q^{2 k}\right)_{\infty}}=: \sum_{n=0}^{\infty} \phi_{n} q^{n}
$$

then $\phi_{k n+r(k-r+1) / 2}$ is always zero.

In [1], Alladi and Gordon generalized the above theorem as follows:

Theorem 1.2. (Alladi and Gordon) Let $1<m<k$ and let $(s, k m)=1$ with $1 \leq s<$ $m k$. Let $r^{*}=(k-1) s$ and $r \equiv r^{*} \bmod m k$ with $1 \leq r<m k$.

Put $r^{\prime}=\left\lceil r^{*} / m k\right\rceil \bmod k$ with $1 \leq r^{\prime}<k$. Write

$$
\frac{\left(q^{r}, q^{m k-r} ; q^{m k}\right)_{\infty}}{\left(q^{s}, q^{m k-s} ; q^{m k}\right)_{\infty}}=: \sum_{n=0}^{\infty} \mu_{n} q^{n} .
$$

Then $\mu_{n}=0$ for $n \equiv r r^{\prime} \bmod k$.

They also proved the following companion result to Theorem 1.2 .

Theorem 1.3. (Alladi and Gordon) Let $m, k, s, r^{*}, r$ and $r^{\prime}$ be defined as in Theorem 1.2 with $k$ odd. Write

$$
\frac{\left(q^{r}, q^{m k-r} ; q^{m k}\right)_{\infty}}{\left(-q^{s},-q^{m k-s} ; q^{m k}\right)_{\infty}}=: \sum_{n=0}^{\infty} \mu_{n}^{\prime} q^{n}
$$

Then $\mu_{n}^{\prime}=0$ for $n \equiv r r^{\prime} \bmod k$.

The result of Alladi and Gordon in Theorem 1.2 does not provide any information about vanishing coefficients in the cases where $k<m$ or $k=m$. In [6], Mc Laughlin proved the following theorem which covers the cases $k \leq m$ as well.

Theorem 1.4. (Mc Laughlin) Let $k>1, m>1$ be positive integers. Let $r=s m+t$, for some integers $s$ and $t$, where $0 \leq s<k, 1 \leq t<m$ and $r$ and $k$ are relatively prime. Let

$$
\frac{\left(q^{r-t k}, q^{m k-(r-t k)} ; q^{m k}\right)_{\infty}}{\left(q^{r}, q^{m k-r} ; q^{m k}\right)_{\infty}}=: \sum_{n=0}^{\infty} \nu_{n} q^{n}
$$

then $\nu_{k n-r s}$ is always zero.

He also found the following companion result to Theorem 1.4 . 
Theorem 1.5. (Mc Laughlin) Let $k>1, m>1$ be positive integers with $k$ odd. Let $r=s m+t$, for some integers $s$ and $t$, where $0 \leq s<k, 1 \leq t<m$ and $r$ and $k$ are relatively prime. Let

$$
\frac{\left(q^{r-t k}, q^{m k-(r-t k)} ; q^{m k}\right)_{\infty}}{\left(-q^{r},-q^{m k-r} ; q^{m k}\right)_{\infty}}=: \sum_{n=0}^{\infty} \nu_{n}^{\prime} q^{n}
$$

then $\nu_{k n-r s}^{\prime}$ is always zero.

All the proofs of the above theorems use Ramanujan's well-known ${ }_{1} \psi_{1}$ summation formula. Very recently, Hirschhorn [5] proved the following interesting result by using only the Jacobi triple product identity and elementary $q$-series manipulations.

Theorem 1.6. (Hirschhorn) If

$$
\sum_{n=0}^{\infty} a_{n} q^{n}:=\left(-q,-q^{4} ; q^{5}\right)_{\infty}\left(q, q^{9} ; q^{10}\right)_{\infty}^{3} \text { and } \sum_{n=0}^{\infty} b_{n} q^{n}:=\left(-q^{2},-q^{3} ; q^{5}\right)_{\infty}\left(q^{3}, q^{7} ; q^{10}\right)_{\infty}^{3}
$$

then

$$
a_{5 n+2}=a_{5 n+4}=0
$$

and

$$
b_{5 n+1}=b_{5 n+4}=0 .
$$

Note that the forms of the $q$-products in Theorem 1.6 are quite different from those in Theorems 1.1 1.5.

Motivated by the work of Hirschhorn [5], Tang [8] found more results on vanishing coefficients in some other comparable $q$-series expansions. In particular, Tang [8] proved the following theorem.

Theorem 1.7. (Tang) If

$$
\sum_{n=0}^{\infty} c_{n} q^{n}:=\left(-q,-q^{4} ; q^{5}\right)_{\infty}^{3}\left(q^{3}, q^{7} ; q^{10}\right)_{\infty} \text { and } \sum_{n=0}^{\infty} d_{n} q^{n}:=\left(-q^{2},-q^{3} ; q^{5}\right)_{\infty}^{3}\left(q, q^{9} ; q^{10}\right)_{\infty}
$$

then

$$
c_{5 n+3}=c_{5 n+4}=0
$$

and

$$
d_{5 n+3}=d_{5 n+4}=0 .
$$

In this paper, we prove the following two theorems. The first theorem clearly implies that instead of proving both (1.1) and (1.2) by Hirschhorn [5], it would have been enough to prove only one of (1.1) or (1.2). Similarly, the second theorem implies that instead of proving both (1.3) and (1.4) by Tang [8], it would have been enough to prove only one of (1.3) or (1.4). 
Theorem 1.8. If

$\sum_{n=0}^{\infty} a_{n} q^{n}:=\left(-q,-q^{4} ; q^{5}\right)_{\infty}\left(q, q^{9} ; q^{10}\right)_{\infty}^{3}$ and $\sum_{n=0}^{\infty} b_{n} q^{n}:=\left(-q^{2},-q^{3} ; q^{5}\right)_{\infty}\left(q^{3}, q^{7} ; q^{10}\right)_{\infty}^{3}$

then

$$
\begin{aligned}
\sum_{n=0}^{\infty} b_{5 n} q^{n} & -\sum_{n=1}^{\infty} a_{5 n-2} q^{n}=\frac{f_{1}^{4}}{f_{2}^{4}} \\
b_{5 n+1} & =a_{5 n-1} \\
b_{5 n+2} & =a_{5 n} \\
b_{5 n+3} & =a_{5 n+1} \\
b_{5 n+4} & =a_{5 n+2} .
\end{aligned}
$$

Theorem 1.9. If

$\sum_{n=0}^{\infty} c_{n} q^{n}:=\left(-q,-q^{4} ; q^{5}\right)_{\infty}^{3}\left(q^{3}, q^{7} ; q^{10}\right)_{\infty}$ and $\sum_{n=0}^{\infty} d_{n} q^{n}:=\left(-q^{2},-q^{3} ; q^{5}\right)_{\infty}^{3}\left(q, q^{9} ; q^{10}\right)_{\infty}$

then

$$
\begin{gathered}
c_{5 n}=d_{5 n}, \\
c_{5 n+2}=d_{5 n+2}, \\
c_{5 n+3}=d_{5 n+3},
\end{gathered}
$$

and

$$
c_{5 n+4}=d_{5 n+4} .
$$

Furthermore,

$$
\sum_{n=0}^{\infty} c_{5 n+1} q^{n}-\sum_{n=0}^{\infty} d_{5 n+1} q^{n}=4 \frac{f_{2}^{4}}{f_{1}^{4}}
$$

which shows that $c_{5 n+1}>d_{5 n+1}$.

By proceeding in a similar way as in Hirschhorn [5], we also prove the following results.

Theorem 1.10. If

$$
\left(\mp q, \mp q^{4} ; q^{5}\right)_{\infty}\left( \pm q^{4}, \pm q^{6} ; q^{10}\right)_{\infty}^{3}=\sum_{n=0}^{\infty} e_{n} q^{n}
$$

and

$$
\left(\mp q^{2}, \mp q^{3} ; q^{5}\right)_{\infty}\left( \pm q^{2}, \pm q^{8} ; q^{10}\right)_{\infty}^{3}=\sum_{n=0}^{\infty} f_{n} q^{n}
$$


where the signs in the products are taken either both upper ones or both lower ones, then

$$
e_{5 n+3}=f_{5 n+4}=0 .
$$

Remark 1.11. The results involving the upper ambiguity signs of Theorem 1.10 have already been proved by Tang [8]. Since our proof works for both the signs, we felt it necessary to keep it here as well.

Theorem 1.12. If

$$
\left(q, q^{4} ; q^{5}\right)_{\infty}\left(-q,-q^{9} ; q^{10}\right)_{\infty}^{3}=\sum_{n=0}^{\infty} g_{n} q^{n}
$$

and

$$
\left(q^{2}, q^{3} ; q^{5}\right)_{\infty}\left(-q^{3},-q^{7} ; q^{10}\right)_{\infty}^{3}=\sum_{n=0}^{\infty} h_{n} q^{n}
$$

then

$$
g_{5 n+2}=h_{5 n+1}=0 .
$$

Theorem 1.13. If

$$
\left(q, q^{4} ; q^{5}\right)_{\infty}\left(q, q^{9} ; q^{10}\right)_{\infty}^{3}=\sum_{n=0}^{\infty} k_{n} q^{n}
$$

and

$$
\left(q^{2}, q^{3} ; q^{5}\right)_{\infty}\left(q^{3}, q^{7} ; q^{10}\right)_{\infty}^{3}=\sum_{n=0}^{\infty} \ell_{n} q^{n}
$$

then

$$
k_{5 n+4}=\ell_{5 n+4}=0 .
$$

Theorem 1.14. If

$$
\left(q, q^{4} ; q^{5}\right)_{\infty}^{3}\left(-q^{3},-q^{7} ; q^{10}\right)_{\infty}=\sum_{n=0}^{\infty} s_{n} q^{n}
$$

and

$$
\left(q^{2}, q^{3} ; q^{5}\right)_{\infty}^{3}\left(-q,-q^{9} ; q^{10}\right)_{\infty}=\sum_{n=0}^{\infty} t_{n} q^{n}
$$

then

$$
s_{5 n+3}=t_{5 n+4}=0 .
$$


Theorem 1.15. If

$$
\left(q, q^{4} ; q^{5}\right)_{\infty}^{3}\left(q^{3}, q^{7} ; q^{10}\right)_{\infty}=\sum_{n=0}^{\infty} u_{n} q^{n}
$$

and

$$
\left(q^{2}, q^{3} ; q^{5}\right)_{\infty}^{3}\left(q, q^{9} ; q^{10}\right)_{\infty}=\sum_{n=0}^{\infty} v_{n} q^{n}
$$

then

$$
u_{5 n+4}=v_{5 n+3}=0 .
$$

We employ simple $q$-series manipulations, Jacobi triple product identity, some preliminary identities for Ramanujan's theta functions, and two known identities for a certain quotient of $q$-products. In Section 2, 4, we prove Theorems 1.8 1.10, respectively. Since the proofs of Theorems $1 . 1 2 \longdiv { 1 . 1 5 }$ are similar in nature, we omit the proofs.

We now end this section by giving some preliminary results that will be used in our proofs. Let $f(a, b)$ denote Ramanuajn's theta function defined by

$$
f(a, b):=\sum_{k=-\infty}^{\infty} a^{k(k+1) / 2} b^{k(k-1) / 2}, \quad|a b|<1 .
$$

Jacobi's famous triple product identity then takes the form

$$
f(a, b)=(-a ; a b)_{\infty}(-b ; a b)_{\infty}(a b ; a b)_{\infty} .
$$

The following preliminary identities easily follow from [4, p. 46, Entry 30].

Lemma 1.16. We have

$$
\begin{aligned}
f\left(a, a b^{2}\right) f\left(b, a^{2} b\right) & =\frac{1}{2} f(1, a b) f(a, b), \\
f(a, b) f(-a,-b) & =f(-a b,-a b) f\left(-a^{2},-b^{2}\right), \\
f(a, b) & =f\left(a^{3} b, a b^{3}\right)+a f\left(\frac{b}{a}, a^{5} b^{3}\right), \\
f^{2}(a, b) & =f\left(a^{2}, b^{2}\right) f(a b, a b)+a f\left(\frac{b}{a}, a^{3} b\right) f\left(1, a^{2} b^{2}\right) .
\end{aligned}
$$

The triple product identity and the identities in the above lemma will be used frequently in our proofs, quite often, without referring.

From [3, Eqs. (1.19) and (1.20)], we also recall the following two identities which will be used in our next two sections.

Lemma 1.17. Let

$$
R(q)=\frac{\left(q, q^{4} ; q^{5}\right)_{\infty}}{\left(q^{2}, q^{3} ; q^{5}\right)_{\infty}}
$$


We have

$$
\frac{1}{R(q) R^{2}\left(q^{2}\right)}-q^{2} R(q) R^{2}\left(q^{2}\right)=\frac{\left(q^{2} ; q^{2}\right)_{\infty}\left(q^{5} ; q^{5}\right)_{\infty}^{5}}{(q ; q)_{\infty}\left(q^{10} ; q^{10}\right)_{\infty}^{5}}
$$

and

$$
\frac{R\left(q^{2}\right)}{R^{2}(q)}-\frac{R^{2}(q)}{R\left(q^{2}\right)}=4 q \frac{\left(q^{10} ; q^{10}\right)_{\infty}^{5}(q ; q)_{\infty}}{\left(q^{5} ; q^{5}\right)_{\infty}\left(q^{2} ; q^{2}\right)_{\infty}}
$$

\section{Proof of Theorem 1.8}

We have

$$
\begin{aligned}
\sum_{n=0}^{\infty} a_{n} q^{n} & =\left(-q,-q^{4} ; q^{5}\right)_{\infty}\left(q, q^{9} ; q^{10}\right)_{\infty}^{3} \\
& =\frac{\left(q^{2}, q^{8} ; q^{10}\right)_{\infty}}{\left(q, q^{4} ; q^{5}\right)_{\infty}} \cdot \frac{\left(q, q^{4}, q^{6}, q^{9} ; q^{10}\right)_{\infty}^{3}}{\left(q^{4}, q^{6} ; q^{10}\right)_{\infty}^{3}} \\
& =\frac{\left(q^{2}, q^{8} ; q^{10}\right)_{\infty}\left(q, q^{4} ; q^{5}\right)_{\infty}^{2}}{\left(q^{4}, q^{6} ; q^{10}\right)_{\infty}^{3}} \\
& =\frac{\left(q, q^{2}, q^{3}, q^{4} ; q^{5}\right)_{\infty}}{\left(q^{2}, q^{4}, q^{6}, q^{8} ; q^{10}\right)_{\infty}} \cdot \frac{\left(q, q^{4} ; q^{5}\right)_{\infty}}{\left(q^{2}, q^{3} ; q^{5}\right)_{\infty}} \cdot \frac{\left(q^{2}, q^{8} ; q^{10}\right)_{\infty}^{2}}{\left(q^{4}, q^{6} ; q^{10}\right)_{\infty}^{2}} \\
& =\frac{(q ; q)_{\infty}\left(q^{10} ; q^{10}\right)_{\infty}}{\left(q^{2} ; q^{2}\right)_{\infty}\left(q^{5} ; q^{5}\right)_{\infty}} \cdot R(q) R^{2}\left(q^{2}\right)
\end{aligned}
$$

and

$$
\begin{aligned}
\sum_{n=0}^{\infty} b_{n} q^{n} & =\left(-q^{2},-q^{3} ; q^{5}\right)_{\infty}\left(q^{3}, q^{7} ; q^{10}\right)_{\infty}^{3} \\
& =\frac{\left(q^{4}, q^{6} ; q^{10}\right)_{\infty}}{\left(q^{2}, q^{3} ; q^{5}\right)_{\infty}} \cdot \frac{\left(q^{2}, q^{3}, q^{7}, q^{8} ; q^{10}\right)_{\infty}^{3}}{\left(q^{2}, q^{8} ; q^{10}\right)_{\infty}^{3}} \\
& =\frac{\left(q^{4}, q^{6} ; q^{10}\right)_{\infty}\left(q^{2}, q^{3} ; q^{5}\right)_{\infty}^{2}}{\left(q^{2}, q^{8} ; q^{10}\right)_{\infty}^{3}} \\
& =\frac{\left(q, q^{2}, q^{3}, q^{4} ; q^{5}\right)_{\infty}}{\left(q^{2}, q^{4}, q^{6}, q^{8} ; q^{10}\right)_{\infty}} \cdot \frac{\left(q^{2}, q^{3} ; q^{5}\right)_{\infty}}{\left(q, q^{4} ; q^{5}\right)_{\infty}} \cdot \frac{\left(q^{4}, q^{6} ; q^{10}\right)_{\infty}^{2}}{\left(q^{2}, q^{8} ; q^{10}\right)_{\infty}^{2}} \\
& =\frac{(q ; q)_{\infty}\left(q^{10} ; q^{10}\right)_{\infty}}{\left(q^{2} ; q^{2}\right)_{\infty}\left(q^{5} ; q^{5}\right)_{\infty}} \cdot \frac{1}{R(q) R^{2}\left(q^{2}\right)}
\end{aligned}
$$

Therefore,

$$
\begin{aligned}
\sum_{n=0}^{\infty} b_{n} q^{n}-\sum_{n=0}^{\infty} a_{n} q^{n+2} & =\frac{(q ; q)_{\infty}\left(q^{10} ; q^{10}\right)_{\infty}}{\left(q^{2} ; q^{2}\right)_{\infty}\left(q^{5} ; q^{5}\right)_{\infty}}\left(\frac{1}{R(q) R^{2}\left(q^{2}\right)}-q^{2} R(q) R^{2}\left(q^{2}\right)\right) \\
& =\frac{(q ; q)_{\infty}\left(q^{10} ; q^{10}\right)_{\infty}}{\left(q^{2} ; q^{2}\right)_{\infty}\left(q^{5} ; q^{5}\right)_{\infty}} \cdot \frac{\left(q^{2} ; q^{2}\right)_{\infty}\left(q^{5} ; q^{5}\right)_{\infty}^{5}}{(q ; q)_{\infty}\left(q^{10} ; q^{10}\right)_{\infty}^{5}} \\
& =\frac{\left(q^{5} ; q^{5}\right)_{\infty}^{4}}{\left(q^{10} ; q^{10}\right)_{\infty}^{4}}
\end{aligned}
$$


Equating the coefficients of $q^{5 n+r}, r=0,1,2,3,4$ from both sides of the above, we readily arrive at (1.5) - (1.9) to finish the proof.

\section{Proof of Theorem $\mathbf{1 . 9}$}

We have

$$
\begin{aligned}
\sum_{n=0}^{\infty} c_{n} q^{n} & =\left(-q,-q^{4} ; q^{5}\right)_{\infty}^{3}\left(q^{3}, q^{7} ; q^{10}\right)_{\infty} \\
& =\frac{\left(q^{2}, q^{8} ; q^{10}\right)_{\infty}^{3}}{\left(q, q^{4} ; q^{5}\right)_{\infty}^{3}} \cdot\left(q^{3}, q^{7} ; q^{10}\right)_{\infty} \\
& =\frac{\left(q^{2}, q^{8} ; q^{10}\right)_{\infty}^{2}\left(q^{2}, q^{3} ; q^{5}\right)_{\infty}}{\left(q, q^{4} ; q^{5}\right)_{\infty}^{3}} \\
& =\frac{\left(q^{2}, q^{4}, q^{6}, q^{8} ; q^{10}\right)_{\infty}}{\left(q, q^{2}, q^{3}, q^{4}\right)_{\infty}} \cdot \frac{\left(q^{2}, q^{3} ; q^{5}\right)_{\infty}^{2}}{\left(q, q^{4} ; q^{5}\right)_{\infty}^{2}} \cdot \frac{\left(q^{2}, q^{8} ; q^{10}\right)_{\infty}}{\left(q^{4}, q^{6} ; q^{10}\right)_{\infty}} \\
& =\frac{\left(q^{5} ; q^{5}\right)_{\infty}\left(q^{2} ; q^{2}\right)_{\infty}}{(q ; q)_{\infty}\left(q^{10} ; q^{10}\right)_{\infty}} \cdot \frac{R\left(q^{2}\right)}{R^{2}(q)}
\end{aligned}
$$

and

$$
\begin{aligned}
\sum_{n=0}^{\infty} d_{n} q^{n} & =\left(-q^{2},-q^{3} ; q^{5}\right)_{\infty}^{3}\left(q, q^{9} ; q^{10}\right)_{\infty} \\
& =\frac{\left(q^{4}, q^{6} ; q^{10}\right)_{\infty}^{3}}{\left(q^{2}, q^{3} ; q^{5}\right)_{\infty}^{3}} \cdot\left(q, q^{9} ; q^{10}\right)_{\infty} \\
& =\frac{\left(q^{4}, q^{6} ; q^{10}\right)_{\infty}^{2}\left(q, q^{4} ; q^{5}\right)_{\infty}}{\left(q^{2}, q^{3} ; q^{5}\right)_{\infty}^{3}} \\
& =\frac{\left(q^{2}, q^{4}, q^{6}, q^{8} ; q^{10}\right)_{\infty}}{\left(q, q^{2}, q^{3}, q^{4}\right)_{\infty}} \cdot \frac{\left(q, q^{4} ; q^{5}\right)_{\infty}^{2}}{\left(q^{2}, q^{3} ; q^{5}\right)_{\infty}^{2}} \cdot \frac{\left(q^{4}, q^{6} ; q^{10}\right)_{\infty}}{\left(q^{2}, q^{8} ; q^{10}\right)_{\infty}} \\
& =\frac{\left(q^{5} ; q^{5}\right)_{\infty}\left(q^{2} ; q^{2}\right)_{\infty}}{(q ; q)_{\infty}\left(q^{10} ; q^{10}\right)_{\infty}} \cdot \frac{R^{2}(q)}{R\left(q^{2}\right)}
\end{aligned}
$$

Therefore,

$$
\begin{aligned}
\sum_{n=0}^{\infty} c_{n} q^{n}-\sum_{n=0}^{\infty} d_{n} q^{n} & =\frac{\left(q^{5} ; q^{5}\right)_{\infty}\left(q^{2} ; q^{2}\right)_{\infty}}{(q ; q)_{\infty}\left(q^{10} ; q^{10}\right)_{\infty}}\left(\frac{R\left(q^{2}\right)}{R^{2}(q)}-\frac{R^{2}(q)}{R\left(q^{2}\right)}\right) \\
& =4 q \frac{\left(q^{5} ; q^{5}\right)_{\infty}\left(q^{2} ; q^{2}\right)_{\infty}}{(q ; q)_{\infty}\left(q^{10} ; q^{10}\right)_{\infty}} \cdot \frac{\left(q^{10} ; q^{10}\right)_{\infty}^{5}(q ; q)_{\infty}}{\left(q^{5} ; q^{5}\right)_{\infty}\left(q^{2} ; q^{2}\right)_{\infty}} \\
& =4 q \frac{\left(q^{10} ; q^{10}\right)_{\infty}^{4}}{\left(q^{5} ; q^{5}\right)_{\infty}^{4}}
\end{aligned}
$$

Equating the coefficients of $q^{5 n+r}, r=0,2,3,4$ from both sides of the above, we have

$$
c_{5 n}=d_{5 n}
$$




$$
\begin{aligned}
\sum_{n=0}^{\infty} c_{5 n+1} q^{n} & -\sum_{n=0}^{\infty} d_{5 n+1} q^{n}=4 \frac{f_{2}^{4}}{f_{1}^{4}} \\
c_{5 n+2} & =d_{5 n+2} \\
c_{5 n+3} & =d_{5 n+3}
\end{aligned}
$$

and

$$
c_{5 n+4}=d_{5 n+4},
$$

which are (1.10) - (1.13). Similarly, extracting the terms involving $q^{5 n+1}$ from both sides of (3.1), diving by $q$, and then replacing $q^{5}$ by $q$, we arrive at (1.14), to complete the proof.

\section{Proof of Theorem $\mathbf{1 . 1 0}$}

Throughout this section, we consider the ambiguity signs in the products to be either all upper ones or all lower ones.

We have

$$
\begin{aligned}
\sum_{n=0}^{\infty} e_{n} q^{n} & =\left(\mp q, \mp q^{4} ; q^{5}\right)_{\infty}\left( \pm q^{4}, \pm q^{6} ; q^{10}\right)_{\infty}^{3} \\
& =\left(\mp q, \mp q^{4}, \pm q^{4}, \pm q^{4}, \pm q^{4}, \mp q^{6}, \pm q^{6}, \pm q^{6}, \pm q^{6}, \mp q^{9} ; q^{10}\right)_{\infty} \\
& =\left(\mp q, \pm q^{4}, \pm q^{6}, \mp q^{9} ; q^{10}\right)_{\infty}\left(q^{8}, q^{12} ; q^{20}\right)_{\infty}\left( \pm q^{4} ; \pm q^{6} ; q^{10}\right)_{\infty} \\
& =U_{1}(q) U_{2} U_{3},
\end{aligned}
$$

where $U_{1}(q)=\left(\mp q, \pm q^{4}, \pm q^{6}, \mp q^{9} ; q^{10}\right)_{\infty}, U_{2}=\left(q^{8}, q^{12} ; q^{20}\right)_{\infty}, U_{3}=\left( \pm q^{4} ; \pm q^{6} ; q^{10}\right)_{\infty}$.

Now,

$$
\begin{aligned}
U_{1}(-q) & =\left( \pm q, \pm q^{4}, \pm q^{6}, \pm q^{9} ; q^{10}\right)_{\infty} \\
& =\left( \pm q, \pm q^{4} ; q^{5}\right)_{\infty} \\
& =\frac{\left( \pm q, \pm q^{4}, q^{5} ; q^{5}\right)_{\infty}}{\left(q^{5} ; q^{5}\right)_{\infty}} \\
& =\frac{1}{\left(q^{5} ; q^{5}\right)_{\infty}} \sum_{m=-\infty}^{\infty}(\mp 1)^{m} q^{\left(5 m^{2}+3 m\right) / 2} \\
& =\frac{1}{\left(q^{5} ; q^{5}\right)_{\infty}}\left(\sum_{m=-\infty}^{\infty} q^{10 m^{2}+3 m} \mp q \sum_{m=-\infty}^{\infty} q^{10 m^{2}+7 m}\right) \\
& =\frac{1}{\left(q^{5} ; q^{10}\right)_{\infty}\left(q^{10} ; q^{10}\right)_{\infty}}\left(\left(-q^{7},-q^{13}, q^{20} ; q^{20}\right)_{\infty} \mp q\left(-q^{3},-q^{17}, q^{20} ; q^{20}\right)_{\infty}\right)
\end{aligned}
$$

and hence,

$$
U_{1}(q)=\frac{\left(q^{5} ; q^{5}\right)_{\infty}\left(q^{20} ; q^{20}\right)_{\infty}}{\left(q^{10} ; q^{10}\right)_{\infty}^{3}}\left(\left(q^{7}, q^{13}, q^{20} ; q^{20}\right)_{\infty} \pm q\left(q^{3}, q^{17}, q^{20} ; q^{20}\right)_{\infty}\right)
$$


Therefore,

$$
\begin{aligned}
& U_{1}(q) U_{2} \\
& =\frac{\left(q^{5} ; q^{5}\right)_{\infty}}{\left(q^{10} ; q^{10}\right)_{\infty}^{3}}\left(q^{8}, q^{12}, q^{20} ; q^{20}\right)\left(\left(q^{7}, q^{13}, q^{20} ; q^{20}\right)_{\infty} \pm q\left(q^{3}, q^{17}, q^{20} ; q^{20}\right)_{\infty}\right) \\
& =\frac{\left(q^{5} ; q^{5}\right)_{\infty}}{\left(q^{10} ; q^{10}\right)_{\infty}^{3}} \sum_{m=-\infty}^{\infty}(-1)^{m} q^{10 m^{2}+2 m} \\
& \times\left(\sum_{n=-\infty}^{\infty}(-1)^{n} q^{10 n^{2}+3 n} \pm q \sum_{n=-\infty}^{\infty}(-1)^{n} q^{10 n^{2}+7 n}\right) \\
& =\frac{\left(q^{5} ; q^{5}\right)_{\infty}}{\left(q^{10} ; q^{10}\right)_{\infty}^{3}}\left(\sum_{m, n=-\infty}^{\infty}(-1)^{m+n} q^{10 m^{2}+2 m+10 n^{2}+3 n}\right. \\
& \left. \pm q \sum_{m, n=-\infty}^{\infty}(-1)^{m+n} q^{10 m^{2}+7 m+10 n^{2}+2 n}\right) \\
& =\frac{\left(q^{5} ; q^{5}\right)_{\infty}}{\left(q^{10} ; q^{10}\right)_{\infty}^{3}}\left(\left(\sum_{r, s=-\infty}^{\infty} q^{10(r+s)^{2}+2(r+s)+10(r-s)^{2}+3(r-s)}\right.\right. \\
& \left.-\sum_{r, s=-\infty}^{\infty} q^{10(r+s-1)^{2}+2(r+s-1)+10(r-s)^{2}+3(r-s)}\right) \\
& \pm q\left(\sum_{r, s=-\infty}^{\infty} q^{10(r+s)^{2}+7(r+s)+10(r-s)^{2}+2(r-s)}\right. \\
& \left.\left.-\sum_{r, s=-\infty}^{\infty} q^{10(r+s-1)^{2}+7(r+s-1)+10(r-s)^{2}+2(r-s)}\right)\right) \\
& =\frac{\left(q^{5} ; q^{5}\right)_{\infty}}{\left(q^{10} ; q^{10}\right)_{\infty}^{3}}\left(\left(\sum_{r, s=-\infty}^{\infty} q^{20 r^{2}+20 s^{2}+5 r+s}-q^{8} \sum_{r, s=-\infty}^{\infty} q^{20 r^{2}+20 s^{2}+15 r+21 s}\right)\right. \\
& \left. \pm q\left(\sum_{r, s=-\infty}^{\infty} q^{20 r^{2}+20 s^{2}+9 r+5 s}-q^{3} \sum_{r, s=-\infty}^{\infty} q^{20 r^{2}+20 s^{2}+11 r+15 s}\right)\right) \\
& =\frac{\left(q^{5} ; q^{5}\right)_{\infty}}{\left(q^{10} ; q^{10}\right)_{\infty}^{3}}\left(\left(-q^{15},-q^{25}, q^{40} ; q^{40}\right)_{\infty}\left(\sum_{n=-\infty}^{\infty} q^{20 n^{2}+n} \pm q \sum_{n=-\infty}^{\infty} q^{20 n^{2}+9 n}\right)\right. \\
& \left.\mp q^{4}\left(-q^{5},-q^{35}, q^{40} ; q^{40}\right)_{\infty}\left(\sum_{n=-\infty}^{\infty} q^{20 n^{2}+11 n} \pm q^{4} \sum_{n=-\infty}^{\infty} q^{20 n^{2}+21 n}\right)\right) .
\end{aligned}
$$

We also have

$$
\begin{aligned}
U_{3} & =\left( \pm q^{4}, \pm q^{6} ; q^{10}\right)_{\infty} \\
& =\frac{1}{\left(q^{10} ; q^{10}\right)_{\infty}}\left( \pm q^{4}, \pm q^{6}, q^{10} ; q^{10}\right)_{\infty}
\end{aligned}
$$




$$
\begin{aligned}
& =\frac{1}{\left(q^{10} ; q^{10}\right)_{\infty}} \sum_{m=-\infty}^{\infty}(\mp 1)^{m} q^{5 m^{2}+m} \\
& =\frac{1}{\left(q^{10} ; q^{10}\right)_{\infty}}\left(\sum_{m=-\infty}^{\infty} q^{20 m^{2}+2 m} \mp q^{4} \sum_{m=-\infty}^{\infty} q^{20 m^{2}+18 m}\right) .
\end{aligned}
$$

It follows that

$$
\begin{aligned}
& \sum_{n=0}^{\infty} e_{n} q^{n} \\
& =\frac{\left(q^{5} ; q^{5}\right)_{\infty}}{\left(q^{10} ; q^{10}\right)_{\infty}^{4}}\left(\left(-q^{15},-q^{25}, q^{40} ; q^{40}\right)_{\infty}\left(\sum_{m=-\infty}^{\infty} q^{20 m^{2}+2 m} \mp q^{4} \sum_{m=-\infty}^{\infty} q^{20 m^{2}+18 m}\right)\right. \\
& \quad \times\left(\sum_{n=-\infty}^{\infty} q^{20 n^{2}+n} \pm q \sum_{n=-\infty}^{\infty} q^{20 n^{2}+9 n}\right) \\
& \quad\left(-q^{5},-q^{35}, q^{40} ; q^{40}\right)_{\infty}\left(\sum_{m=-\infty}^{\infty} q^{20 m^{2}+2 m} \mp q^{4} \sum_{m=-\infty}^{\infty} q^{20 m^{2}+18 m}\right) \\
& \left.\quad \times\left(q^{4} \sum_{n=-\infty}^{\infty} q^{20 n^{2}+11 n} \pm q^{8} \sum_{n=-\infty}^{\infty} q^{20 n^{2}+21 n}\right)\right) \\
& =\frac{\left(q^{5} ; q^{5}\right)_{\infty}\left(\left(-q^{15},-q^{25}, q^{40} ; q^{40}\right)_{\infty}\left(S_{1} \mp S_{2} \pm S_{3}-S_{4}\right)\right.}{\left(q^{10} ; q^{10}\right)_{\infty}^{4}} \\
& \left.\quad \mp\left(-q^{5},-q^{35}, q^{40} ; q^{40}\right)_{\infty}\left(S_{5} \mp S_{6} \pm S_{7}-S_{8}\right)\right),
\end{aligned}
$$

where

$$
\begin{aligned}
& S_{1}=\sum_{m, n=-\infty}^{\infty} q^{20 m^{2}+20 n^{2}+2 m+n}, \quad S_{2}=q^{4} \sum_{m, n=-\infty}^{\infty} q^{20 m^{2}+20 n^{2}+18 m+n}, \\
& S_{3}=q \sum_{m, n=-\infty}^{\infty} q^{20 m^{2}+20 n^{2}+2 m+9 n}, \quad S_{4}=q^{5} \sum_{m, n=-\infty}^{\infty} q^{20 m^{2}+20 n^{2}+18 m+9 n} \\
& S_{5}=q^{4} \sum_{m, n=-\infty}^{\infty} q^{20 m^{2}+20 n^{2}+2 m+11 n}, \quad S_{6}=q^{8} \sum_{m, n=-\infty}^{\infty} q^{20 m^{2}+20 n^{2}+18 m+11 n} \\
& S_{7}=q^{8} \sum_{m, n=-\infty}^{\infty} q^{20 m^{2}+20 n^{2}+2 m+21 n}, \quad S_{8}=q^{12} \sum_{m, n=-\infty}^{\infty} q^{20 m^{2}+20 n^{2}+18 m+21 n} .
\end{aligned}
$$

Proceeding as in [5], it can be shown that the 3-components of the sums $S_{1}, S_{2}, \ldots, S_{8}$ are, respectively,

$$
q^{43} \sum_{r, s=-\infty}^{\infty} q^{100 r^{2}+100 s^{2}+125 r+40 s}, \quad q^{23} \sum_{r, s=-\infty}^{\infty} q^{100 r^{2}+100 s^{2}+75 r+60 s},
$$




$$
\begin{array}{ll}
q^{23} \sum_{r, s=-\infty}^{\infty} q^{100 r^{2}+100 s^{2}+75 r+60 s}, & q^{43} \sum_{r, s=-\infty}^{\infty} q^{100 r^{2}+100 s^{2}+125 r+40 s}, \\
q^{13} \sum_{r, s=-\infty}^{\infty} q^{100 r^{2}+100 s^{2}+25 r+60 s}, & q^{8} \sum_{r, s=-\infty}^{\infty} q^{100 r^{2}+100 s^{2}+25 r+40 s}, \\
q^{8} \sum_{r, s=-\infty}^{\infty} q^{100 r^{2}+100 s^{2}+25 r+40 s}, & q^{13} \sum_{r, s=-\infty}^{\infty} q^{100 r^{2}+100 s^{2}+25 r+60 s} .
\end{array}
$$

Since these cancel in pairs, we conclude that $e_{5 n+3}=0$.

Similarly, we have

$$
\begin{aligned}
\sum_{n=0}^{\infty} f_{n} q^{n} & =\left(\mp q^{2}, \mp q^{3} ; q^{5}\right)_{\infty}\left( \pm q^{2}, \pm q^{8} ; q^{10}\right)_{\infty}^{3} \\
& =\left(\mp q^{2}, \pm q^{2}, \pm q^{2}, \pm q^{2}, \mp q^{3} \mp q^{7}, \mp q^{8} \pm q^{8}, \pm q^{8}, \pm q^{8} ; q^{10}\right)_{\infty} \\
& =\left( \pm q^{2}, \mp q^{3}, \mp q^{7}, \pm q^{8} ; q^{10}\right)_{\infty}\left(q^{4}, q^{16} ; q^{20}\right)_{\infty}\left( \pm q^{2} ; \pm q^{8} ; q^{10}\right)_{\infty} \\
& =V_{1}(q) V_{2} V_{3},
\end{aligned}
$$

where $V_{1}(q)=\left( \pm q^{2}, \mp q^{3}, \mp q^{7}, \pm q^{8} ; q^{10}\right)_{\infty}, V_{2}=\left(q^{4}, q^{16} ; q^{20}\right)_{\infty}, V_{3}=\left( \pm q^{2} ; \pm q^{8} ; q^{10}\right)_{\infty}$.

Now

$$
\begin{aligned}
V_{1}(-q) & =\left( \pm q^{2}, \pm q^{3}, \pm q^{7}, \pm q^{8} ; q^{10}\right)_{\infty} \\
& =\left( \pm q^{2}, \pm q^{3} ; q^{5}\right)_{\infty} \\
& =\frac{\left( \pm q^{2}, \pm q^{3}, q^{5} ; q^{5}\right)_{\infty}}{\left(q^{5} ; q^{5}\right)_{\infty}} \\
& =\frac{1}{\left(q^{5} ; q^{5}\right)_{\infty}} \sum_{m=-\infty}^{\infty}(\mp 1)^{m} q^{\left(5 m^{2}+m\right) / 2} \\
& =\frac{1}{\left(q^{5} ; q^{5}\right)_{\infty}}\left(\sum_{m=-\infty}^{\infty} q^{10 m^{2}+m} \mp q^{2} \sum_{m=-\infty}^{\infty} q^{10 m^{2}+9 m}\right) \\
& =\frac{1}{\left(q^{5} ; q^{10}\right)_{\infty}\left(q^{10} ; q^{10}\right)_{\infty}}\left(\left(-q^{9},-q^{11}, q^{20} ; q^{20}\right)_{\infty} \mp q^{2}\left(-q,-q^{19}, q^{20} ; q^{20}\right)_{\infty}\right) .
\end{aligned}
$$

Therefore,

$$
V_{1}(q)=\frac{\left(q^{5} ; q^{5}\right)_{\infty}\left(q^{20} ; q^{20}\right)_{\infty}}{\left(q^{10} ; q^{10}\right)_{\infty}^{3}}\left(\left(q^{9}, q^{11}, q^{20} ; q^{20}\right)_{\infty} \mp q^{2}\left(q, q^{19}, q^{20} ; q^{20}\right)_{\infty}\right),
$$

and hence,

$$
\begin{aligned}
& V_{1}(q) V_{2} \\
& =\frac{\left(q^{5} ; q^{5}\right)_{\infty}}{\left(q^{10} ; q^{10}\right)_{\infty}^{3}}\left(q^{4}, q^{16}, q^{20} ; q^{20}\right)\left(\left(q^{9}, q^{11}, q^{20} ; q^{20}\right)_{\infty} \mp q^{2}\left(q, q^{19}, q^{20} ; q^{20}\right)_{\infty}\right) \\
& =\frac{\left(q^{5} ; q^{5}\right)_{\infty}}{\left(q^{10} ; q^{10}\right)_{\infty}^{3}} \sum_{m=-\infty}^{\infty}(-1)^{m} q^{10 m^{2}+6 m}\left(\sum_{n=-\infty}^{\infty}(-1)^{n} q^{10 n^{2}+n}\right.
\end{aligned}
$$




$$
\begin{aligned}
& \left.\mp q^{2} \sum_{n=-\infty}^{\infty}(-1)^{n} q^{10 n^{2}+9 n}\right) \\
& =\frac{\left(q^{5} ; q^{5}\right)_{\infty}}{\left(q^{10} ; q^{10}\right)_{\infty}^{3}}\left(\sum_{m, n=-\infty}^{\infty}(-1)^{m+n} q^{10 m^{2}+6 m+10 n^{2}+n}\right. \\
& \left.\mp q^{2} \sum_{m, n=-\infty}^{\infty}(-1)^{m+n} q^{10 m^{2}+9 m+10 n^{2}+6 n}\right) \\
& =\frac{\left(q^{5} ; q^{5}\right)_{\infty}}{\left(q^{10} ; q^{10}\right)_{\infty}^{3}}\left(\left(\sum_{r, s=-\infty}^{\infty} q^{10(r+s)^{2}+6(r+s)+10(r-s)^{2}+(r-s)}\right.\right. \\
& \left.-\sum_{r, s=-\infty}^{\infty} q^{10(r+s-1)^{2}+6(r+s-1)+10(r-s)^{2}+(r-s)}\right) \\
& \mp q^{2}\left(\sum_{r, s=-\infty}^{\infty} q^{10(r+s)^{2}+9(r+s)+10(r-s)^{2}+6(r-s)}\right. \\
& \left.\left.-\sum_{r, s=-\infty}^{\infty} q^{10(r+s-1)^{2}+9(r+s-1)+10(r-s)^{2}+6(r-s)}\right)\right) \\
& =\frac{\left(q^{5} ; q^{5}\right)_{\infty}}{\left(q^{10} ; q^{10}\right)_{\infty}^{3}}\left(\left(\sum_{r, s=-\infty}^{\infty} q^{20 r^{2}+20 s^{2}+7 r+5 s}-q^{4} \sum_{r, s=-\infty}^{\infty} q^{20 r^{2}+20 s^{2}+13 r+15 s}\right)\right. \\
& \left.\mp q^{2}\left(\sum_{r, s=-\infty}^{\infty} q^{20 r^{2}+20 s^{2}+15 r+3 s}-q \sum_{r, s=-\infty}^{\infty} q^{20 r^{2}+20 s^{2}+5 r+17 s}\right)\right) \\
& =\frac{\left(q^{5} ; q^{5}\right)_{\infty}}{\left(q^{10} ; q^{10}\right)_{\infty}^{3}}\left(\left(-q^{15},-q^{25}, q^{40} ; q^{40}\right)_{\infty}\left(\sum_{n=-\infty}^{\infty} q^{20 n^{2}+7 n} \pm q^{3} \sum_{n=-\infty}^{\infty} q^{20 n^{2}+17 n}\right)\right. \\
& \left.\mp\left(-q^{5},-q^{35}, q^{40} ; q^{40}\right)_{\infty}\left(q^{2} \sum_{n=-\infty}^{\infty} q^{20 n^{2}+3 n} \pm q^{4} \sum_{n=-\infty}^{\infty} q^{20 n^{2}+13 n}\right)\right) .
\end{aligned}
$$

Also,

$$
\begin{aligned}
V_{3}(q) & =\left( \pm q^{2}, \pm q^{8} ; q^{10}\right)_{\infty} \\
& =\frac{1}{\left(q^{10} ; q^{10}\right)_{\infty}}\left( \pm q^{2}, \pm q^{8}, q^{10} ; q^{10}\right)_{\infty} \\
& =\frac{1}{\left(q^{10} ; q^{10}\right)_{\infty}} \sum_{m=-\infty}^{\infty}(\mp 1)^{m} q^{5 m^{2}+3 m} \\
& =\frac{1}{\left(q^{10} ; q^{10}\right)_{\infty}}\left(\sum_{m=-\infty}^{\infty} q^{20 m^{2}+6 m} \mp q^{2} \sum_{m=-\infty}^{\infty} q^{20 m^{2}+14 m}\right) .
\end{aligned}
$$


It follows that

$$
\begin{aligned}
\sum_{n=0}^{\infty} f_{n} q^{n} \\
=\frac{\left(q^{5} ; q^{5}\right)_{\infty}}{\left(q^{10} ; q^{10}\right)_{\infty}^{4}}\left(\left(-q^{15},-q^{25}, q^{40} ; q^{40}\right)_{\infty}\left(\sum_{m=-\infty}^{\infty} q^{20 m^{2}+6 m} \mp q^{2} \sum_{m=-\infty}^{\infty} q^{20 m^{2}+14 m}\right)\right. \\
\quad \times\left(\sum_{n=-\infty}^{\infty} q^{20 n^{2}+7 n} \pm q^{3} \sum_{n=-\infty}^{\infty} q^{20 n^{2}+17 n}\right) \\
\mp\left(-q^{5},-q^{35}, q^{40} ; q^{40}\right)_{\infty}\left(\sum_{m=-\infty}^{\infty} q^{20 m^{2}+6 m} \mp q^{2} \sum_{m=-\infty}^{\infty} q^{20 m^{2}+14 m}\right) \\
\left.\quad \times\left(q^{2} \sum_{n=-\infty}^{\infty} q^{20 n^{2}+3 n} \pm q^{4} \sum_{n=-\infty}^{\infty} q^{20 n^{2}+13 n}\right)\right) \\
\left.=\frac{\left(q^{5} ; q^{5}\right)_{\infty}\left(\left(-q^{15},-q^{25}, q^{40} ; q^{40}\right)_{\infty}\left(T_{1} \mp T_{2} \pm T_{3}-T_{4}\right)\right.}{\left(q^{10} ; q^{10}\right)_{\infty}^{4}}\left(-q^{5},-q^{35}, q^{40} ; q^{40}\right)_{\infty}\left(T_{5} \mp T_{6} \pm T_{7}-T_{8}\right)\right) \\
\quad \mp
\end{aligned}
$$

where

$$
\begin{aligned}
& T_{1}=\sum_{m, n=-\infty}^{\infty} q^{20 m^{2}+20 n^{2}+6 m+7 n}, \quad T_{2}=q^{2} \sum_{m, n=-\infty}^{\infty} q^{20 m^{2}+20 n^{2}+14 m+7 n}, \\
& T_{3}=q^{3} \sum_{m, n=-\infty}^{\infty} q^{20 m^{2}+20 n^{2}+6 m+17 n}, \quad T_{4}=q^{5} \sum_{m, n=-\infty}^{\infty} q^{20 m^{2}+20 n^{2}+14 m+17 n}, \\
& T_{5}=q^{2} \sum_{m, n=-\infty}^{\infty} q^{20 m^{2}+20 n^{2}+6 m+3 n}, \quad T_{6}=q^{4} \sum_{m, n=-\infty}^{\infty} q^{20 m^{2}+20 n^{2}+14 m+3 n}, \\
& T_{7}=q^{4} \sum_{m, n=-\infty}^{\infty} q^{20 m^{2}+20 n^{2}+6 m+13 n}, \quad T_{8}=q^{6} \sum_{m, n=-\infty}^{\infty} q^{20 m^{2}+20 n^{2}+14 m+13 n} .
\end{aligned}
$$

It can be shown that, the 4 -components of the sums $T_{1}, T_{2}, \ldots, T_{8}$ are, respectively,

$$
\begin{array}{ll}
q^{14} \sum_{r, s=-\infty}^{\infty} q^{100 r^{2}+100 s^{2}+20 r+75 s}, & q^{29} \sum_{r, s=-\infty}^{\infty} q^{100 r^{2}+100 s^{2}+80 r+75 s}, \\
q^{29} \sum_{r, s=-\infty}^{\infty} q^{100 r^{2}+100 s^{2}+80 r+75 s}, & q^{14} \sum_{r, s=-\infty}^{\infty} q^{100 r^{2}+100 s^{2}+20 r+75 s}, \\
q^{19} \sum_{r, s=-\infty}^{\infty} q^{100 r^{2}+100 s^{2}+80 r+25 s}, & q^{4} \sum_{r, s=-\infty}^{\infty} q^{100 r^{2}+100 s^{2}+20 r+25 s}, \\
q^{4} \sum_{r, s=-\infty}^{\infty} q^{100 r^{2}+100 s^{2}+20 r+25 s}, & q^{19} \sum_{r, s=-\infty}^{\infty} q^{100 r^{2}+100 s^{2}+80 r+25 s},
\end{array}
$$


and these cancel in pairs. Therefore, we arrive at $f_{5 n+4}=0$ to finish the proof.

\section{ACKNOWLEDGMENT}

The authors would like to thank the anonymous referee for his/her comments. The

first author's research was partially supported by Grant no. MTR/2018/000157 of Science \& Engineering Research Board (SERB), DST, Government of India.

\section{REFERENCES}

[1] Alladi, K., Gordon, B.: Vanishing Coefficients in the Expansion of Products of Rogers-Ramanujan Type. In: The Rademacher Legacy in Mathematics(University Park, PA, 1992), Contemporary Mathematics, 166 (American Mathematical Society, Providence, RI, 1994), 129-139 (1994)

[2] Andrews, G.E., Bressoud, D.: Vanishing coefficients in infinite product expansions. J. Aust. Math. Soc. Ser. A 27, 199-202 (1979)

[3] Baruah, N.D., Begum, N.M.: Exact generating functions for the number of partitions into distinct parts. Int. J. Number Theory 14, 1995-2011 (2018)

[4] Berndt, B.C.: Ramanujan's Notebooks, Part III. Springer, New York (1991)

[5] Hirschhorn, M.D.: Two remarkable $q$-series expansions. Ramanujan J. https://doi.org/ 10.1007/ s11139-018-0016-9 (2018)

[6] Mc Laughlin, J.: Further results on vanishing coefficients in infinite product expansions. J. Aust. Math. Soc. Ser. A 98, 69-77 (2015)

[7] Richmond, B., Szekeres, G.: The Taylor coefficients of certain infinite products. Acta Sci. Math. (Szeged) 40, 347-369 (1978)

[8] Tang, D.: Vanishing coefficients in some $q$-series expansions. Int. J. Number Theory https://doi.org/10.1142/S1793042119500398 (2018)

Department of Mathematical Sciences, Tezpur University, Napaam-784028, SonitPUR, ASSAM, INDIA

E-mail address: nayan@tezu.ernet.in

Department of Mathematical Sciences, Tezpur University, Napaam-784028, SonitPUR, ASSAM, INDIA

E-mail address: mandeep@tezu.ernet.in 